\title{
Multiphoton Confocal Microscopy (in vivo Imaging) in the Study of Early Response of Macrophages/Microglia in Damaged Midbrain of Juvenile Chum Salmon Oncorhynchus keta
}

\author{
Pushchina E. V. ${ }^{1, ~}$, Varaksin A. A. ${ }^{1}$, Shukla S. ${ }^{2}$, Bulygyn D. A. ${ }^{3}$ \\ ${ }^{1}$ Laboratory of Cytophysiology, A.V. Zhirmunsky Institute of Marine Biology Far Eastern Branch of Russian Academy of Sciences, \\ Vladivostok, Russia \\ ${ }^{2}$ Brien Holden Eye Research Centre, L.V. Prasad Eye Institute, Hyderabad, India \\ ${ }^{3}$ Department of Cell Biology and Genetics, Far Eastern Federal University, Vladivostok, Russia
}

Email address:

puschina@mail.ru (Pushchina E. V.)

\section{To cite this article:}

Pushchina E. V., Varaksin A. A., Shukla S., Bulygyn D. A.. Multiphoton Confocal Microscopy (in vivo Imaging) in the Study of Early Response of Macrophages/Microglia in Damaged Midbrain of Juvenile Chum Salmon Oncorhynchus keta. American Journal of BioScience. Special Issue: Adult and Reparative Neurogenesis: Actual Questions. Vol. 3, No. 2-3, 2015, pp. 12-18. doi: 10.11648/j.ajbio.s.2015030203.12

\begin{abstract}
We used multiphoton confocal microscopy for the in vivo study of early response of macrophages/microglia in the damaged midbrain of juvenile chum salmon Oncorhynchus keta. The results obtained allow the use of injection of DiI in the area of brain injury as a method to identify a population of phagocytic cells in the brain, based on the physiological response of macrophages/microglia. Thus, the injury with injection of small particles of dye DiI causes the phagocytic response from macrophages within a 30 minutes after the application of the damaging effects. This allows the use of DiI as a vital nonspecific marker of macrophages/microglia. It can be regarded as an effective method of identifying populations of phagocytic cells in the brain, as the effective molecular markers that allow selective identification of populations of macrophages and microglia in the brain of the fish have not been developed so far. We supposed that using multiphoton confocal microscopy in vivo experiments allow to have the substantial preference. Damage to living cells decreases photo induced processes, because of the much lower absorption of tissues and cells in the infrared region as compared with the ultraviolet one. For this reason, it provides more depth of penetration in biological objects (animal). The main beneficial consequence of this is the better survival of a biological object with good image quality.
\end{abstract}

Keywords: Carbocyanine Dye DiI, In Vivo Imaging, Multiphoton Microscopy, Neuronal Tracing Technique, Transneuronal Transport, Macrophages, Microglia

\section{Introduction}

It is known that among other vertebrates, fish are able to effectively restore the structure of cells and fibers after CNS injury. One of the proposed mechanisms of successful regeneration of nervous tissue of fish brain is an early response of microglia that determines the efficiency of elimination of damaged cells by apoptosis [1]. Being a convenient model for neurogenic study the animals from evolutionarily ancient groups are increasingly being used; in their brain are a large number of periventricular neurogenic zones and active zones of secondary neurogenesis $[1,2]$. After a damaging effect, processes of regeneration in the brain of fish is determined by several factors, differ from other vertebrates, including mammals and humans [3, 4].

It is known that after brain injury in mammals develops a number of pathological changes associated with inflammatory response, preventing a quick brain repair. The primary inflammatory process is formed as a result of the toxic effects of glutamate and other inflammatory mediators, as well as subsequent pathological changes associated with the development of secondary inflammation and is accompanied by the loss of a large number of cells $[5,6]$. It is shown that as a result of CNS trauma large number of mammalian cells undergo necrosis and only a small proportion of the cells is eliminated by apoptosis [7]. In the 
brain of fish after traumatic injury cell response develops in a different scenario; after 5 minutes in the damaged cells develop apoptotic response, which increases over the next few days [8]. Elimination of damaged cells is carried out with the participation of phagocytes (microglia/macrophages). It is extremely effective to dispose of damaged cells, providing a "clean death" of cells, without leaving a damaged cellular material, allowing the development of secondary inflammation [9]. Filling a large number of cell loss as a result of fish brain damage may occur from different sources: radial glia, centers of primary and secondary proliferation, as well as neurogenic niches. High regenerative potential in CNS of fish is provided by both the activation of specific regeneration-associating factors [10] and the influence of factors affecting the neuroprotection (carrying out the protection of damaged cells and providing long-term survival of cells formed as a result of reparative neurogenesis).

The aim of this work was to study the early in vivo response of macrophages/microglia in damaged midbrain of juvenile chum salmon Oncorhynchus keta using multifoton confocal microscopy.

\section{Materials and Methods}

\subsection{Experimental Animals}

The experiments were performed on 10 three-month-old juveniles of chum salmon Oncorhynchus keta. Average body weight of juveniles was $8-10 \mathrm{~g}$ and body length was $3-3.5 \mathrm{~cm}$. The animals were obtained from Ryasan local breeding hatchery (Prymorsky region, Russia) in year 2014. For adaptation the young fish were kept in aerated aquarium with fresh water at temperature $15-16^{\circ} \mathrm{C}$. The length of the photoperiod (the ratio of the illuminated and dark period in days) was 14/10 hours. The animals were kept on 1 time diet. The amount of dissolved oxygen in water was 7-10 mg/liter that correspond to normal oxygen saturation.

All experimental manipulations with animals were performed in accordance with the rules governed by the charter of Institute of Marine Biology, Resource Center IMB FEB RAS and the Ethics Committee, which regulates the humane treatment with experimental animals. Animals were anesthetized in a solution of tricaine methane-sulfonate MS222 (Sigma, USA) for 3-5 min.

\subsection{Application of Damaging Effect}

In our study was used fluorescent lipophilic carbocyanine dye DiI (1,1'-dioctadecyl-3,3,3'3'tetramethylindocarbocyanine perchlorate, Aldrich, Sigma, USA) (Fig. 1). The dye (DiI) is purple violet crystals; the spectra of this dye show an absorption maximum at $549 \mathrm{~nm}$, and an emission maximum of $565 \mathrm{~nm}$ [11]. It is mildly fluorescent in aqueous suspension, but becomes bright when it binds to cell membrane. Once bound to cell membrane it diffuses laterally in two dimensions; if there is no diffusion barrier, DiI generally stains the whole leaflet (one surface) of a biological membrane rapidly, but does not readily flip across to the other leaflet.

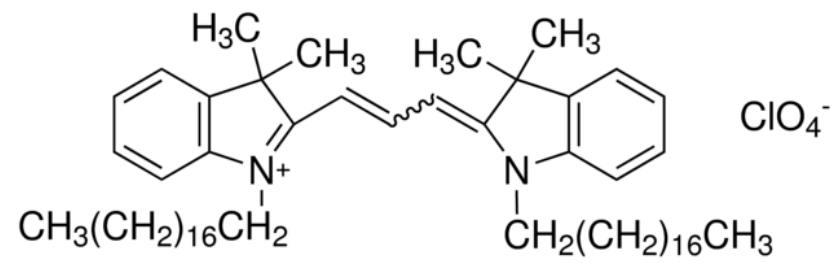

Figure 1. The lipophilic tracer 1,1'-dioctadecyl-3,3,3'3'tetramethylindocarbocyanine perchlorate (DiI) fluorescent dye.

In mesencephalon (tectum opticum) of fish thin needle containing crystals of dye puncture to a depth of 2-3 mm was applied. After that the animal was placed in a separate aquarium with fresh water and with enhanced aeration for recovery. Observation of the animals was performed for 30 minutes or 2 days, then the animal was placed in a special chamber of open cultivation POC-R (Carl Zeiss, Germany), with the aquarium water. POC-R chamber has observation area $6.6 \mathrm{~cm}^{2}, \varnothing 29 \mathrm{~mm}$ and weight $39 \mathrm{~g}$ (Fig. 2). The immobilized animal was turned onto back and surrounded by a damp cloth after using MS-222.

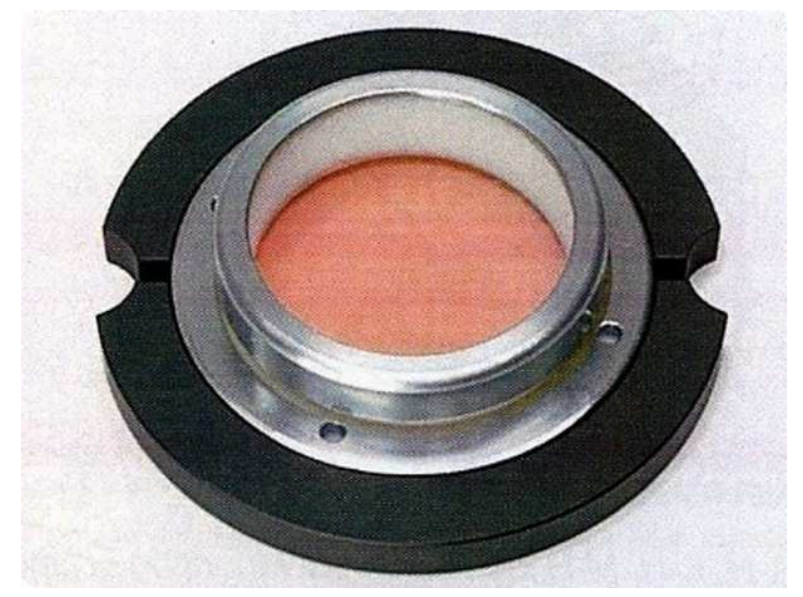

Figure 2. The chamber of open cultivation POC-R (Carl Zeiss, Germany). In $P O C-R$ chamber (observation area $6.6 \mathrm{~cm}^{2}, \varnothing 29 \mathrm{~mm}$; weight $39 \mathrm{~g}$ ) was carried out in vivo monitoring after injury of midbrain of 3-month-old juvenile chum salmon Oncorhynchus keta.

\subsection{Multiphoton Confocal Microscopy}

For in vivo monitoring was used multiphoton confocal microscope 780 LSM (Carl Zeiss, Germany). This type of microscopy is based on the nonlinear optical effect in which the increased power density of the light increases the probability of absorption of fluorochrome atom at the same time with two or more photons. After that, the atom emits photon energy larger than the one-photon absorption. Since the wavelength of the radiation is inversely proportional to the energy of the photons emitted from the fluorochrome, the emission wavelength is a shorter than the excitation wavelength [12]. Multiphoton absorption is possible only at very high power densities. Such power density is achieved both due to focusing of laser radiation and by reducing the duration of the laser pulse. In 780 LSM microscope for 
multiphoton microscopy, we used lasers with pulse durations up to 10-13 (100 femtoseconds). Pulses follow with a high frequency $(100 \mathrm{MHz})$, and the intervals between pulses is significantly shorter than the time ranking of the beam during scanning. The average radiation power at the same time may be small, of the same order as that of a single-photon excitation.

\subsection{Monitoring in Vivo DiI-Labeled Cells}

Animal at the beginning of the experiment was lying on the back, was submerged in the aquarium water, the surface of the skull was tightly pressed to the bottom wall of the POC-R chamber. For the study we used confocal microscope LSM 780 in multiphotonic laser mode with an inverted lens. The brain of animal was examined as a whole, without opening the skull and removing pigmented primary brain tunic. Thus, the substance for the study of DiI-labeled cells initially represented structurally heterogeneous environment, including the bones of the skull, cerebro-spinal fluid, primary brain tunic and brain tissue.

The observation was carried out with special planar lens with built-in color correction (Advanced Correction System) at 20x magnification. The sample of cells was carried out in the middle portion of the optical section at a depth of $200 \mu \mathrm{m}$ (Fig. 3A). Since the observations were made in in vivo mode (without production of brain sections), the scanning process have some aberrations. We excluded from our observations cells located in the peripheral portion of the optical section. Optical sections located at a depth of $200 \mu \mathrm{m}$ have been used for quantitative evaluation of morphological parameters and densitometry. The thickness of the optical section was $20 \mu \mathrm{m}$.

\subsection{Densitometry}

Measurement of the optical density was performed using software of laser confocal microscope 780 LSM (Carl Zeiss, Germany). Densitometric evaluation of the optical density was measured in the range from 0 to 170 units of optical density (UOD). The value of the optical density of all cells set in a range from 23.88 to 92.50 UOD. The most intensely labeled cells had an optical density in the range of 70.08 to 92.5 UOD; average OD was recorded in the range 50.8869.88 UOD, and least intense of DiI-labeled cells fluoresce was in the range 23.88-49.66 UOD.

LSM files have been converted to a format JEPG. Voluminous files, which are mounted in a single file gallery of optical sections, divided into several fragments. Each fragment contains heterogeneous portions containing labeled cells and background areas, which differ in optical density parameters. 10 most detailed optical sections used for creation of $3 \mathrm{D}$ reconstruction (Fig. 3F).

\subsection{Statistical Analysis}

Quantitative morphometric data processing was carried out using the program Statistica 12 and Microsoft Excel 2010. Data are presented as mean \pm SEM. To assess the degree of correlation between morphometric parameters and the optical density of DiI-labeled cells was used correlative analysis and calculated the Pearson coefficient.

\section{Results}

After a $30 \mathrm{~min}$ of exposure DiI to mesencephalon of juvenils $O$. keta we observed local bright fluorescent cell bodies located in the midbrain tegmentum (Fig. 3A). Cells were numerous, uniformly distributed on the depth of the investigated optical section and formed clearly a visible row of selective labeled components (Fig. 3A). Intensity of DiIlabeling was not the same in different cells; the parameters of the optical density (OD) in DiI labeled cells ranged from 23.88 UOD (minimum) to 92.5 UOD (maximum). We found 2 day after injury that distribution of DiI-labeled cells in different optical section was unevenly. To recreate an objective picture was used specialized optics (planar, align lens with magnification 20x), allowing to obtain the best possible image on a live animal with in vivo monitoring and summarize data about distribution of cells on multiple optical sections. In this case, peripheral aberration formed due to non-planar sample study was completely leveled.

\subsection{Morphological Characteristics and Densitometry of DiI-Labeled Cells 30 min After Injury}

As a result of optical scanning we observed DiI-labeled elements without outgrowths, which formed local clusters (after 2 days) and were presented by individual elements (after $30 \mathrm{~min}$ ) (Fig. 3C, D). Our observation showed that labeled cells are distributed like row (Fig. 3A), transverse diameter of which was $100-120 \mu \mathrm{m}$. In this row were identified cells, morphometric characteristics are given in Table. After measurements we identified 4 major types of cells.

Investigation of optical sections showed the presence of large numbers of small, intensely fluorescent granules whose sizes ranged from 2.44 to $3.51 \mu \mathrm{m}$. The average optical density of such elements was $57.7 \pm 14.2$ UOD (Table). This group of elements has been attributed to 1st type of noncellular elements representing, presumably, the individual pellets of dye contained in injury site. The proportion of such elements in tested optical sections was $13.4 \%$ (Table).

According to Candal with coauthors [13], the minimal size of cells in the brain of juvenile salmonids is 3.5-4.0 $\mu \mathrm{m}$. According to this classification and the results of their own measurements morphometric parameters of cells of 2 nd type in tegmentum of chum salmon ranged from 3.57-5.98 $\mu \mathrm{m}$ (Table). This type includes rounded or slightly elongated shaped cells without outgrowths. 2nd types of cells represented the largest group, 67\% (Table). This cell type was predominant among all others in most of the optical sections. Maximal accumulation of cells of 2 nd type, we observed in the central part, corresponding to lesion area (Fig. 3C). The cells were placed singly, but often they formed conglomerates with several elements (Fig. 3C). 3rd type of DiI-labeled elements was represented by larger cells with dimensions $10.8 \pm 1.7 / 7.7 \pm 0.9 \mu \mathrm{m}$ (Table). Cells of this 
type have no outgrowths and have round or oval shape (Fig. 3D). Compared to cells of 2nd type, the cells of 3rd type had a higher intensity of fluorescence $67 \pm 11.1$ UOD (Table). Cells of 4th type were the largest elements, their size was $19.1 \pm 2.2 / 11.5 \pm 1.9 \mu \mathrm{m}$; they accounted for a smaller portion (3.7\%) of the DiI-labeled cells (Table). Often these cells have been grouped in small clusters for 4-6 elements (Fig. 3D). In the cells of 4th type was found less intense fluorescence $52.4 \pm 9.2$ UOD (Table).

Table. Morphometric, densitometric parameters and number of DiI-labeled cells within 30 min after injury of midbrain of juveniles Chum salmon Oncorhynchus keta

\begin{tabular}{|c|c|c|c|c|}
\hline Types of cell & $\begin{array}{l}\text { Large diameter of cells, } \\
(\mu \mathrm{m})\end{array}$ & $\begin{array}{l}\text { Small diameter of cells, } \\
(\mu \mathrm{m})\end{array}$ & Number of cells, (\%) & Optical density, (UOD) \\
\hline 1 (noncellular material) & $3.20 \pm 0.34$ & $2.71 \pm 0.38$ & 13.4 & $57.7 \pm 14.2$ \\
\hline 3 & $10.84 \pm 1.76$ & $7.72 \pm 0.99$ & 15.7 & $67.0 \pm 11.1$ \\
\hline 4 & $19.16 \pm 2.21$ & $11.58 \pm 1.94$ & 3.7 & $52.4 \pm 9.2$ \\
\hline
\end{tabular}

\subsection{Overlay of Transmitted and Fluorescent Channels for the Study of Surface and Deeper Layers of Brain In Vivo 2 Days After Injury}

After 2 days in the optical sections of damaged tegmentum, the density of distribution of DiI-labeled cells was demonstrated as occurrence of cell conglomerates (Fig. 3B). To investigate the space relationships of DiI-labeled cell conglomerates observed in the area of injury 2 days after injury with DiI-unlabelad, but intensely pigmented melanocytes of primary brain tunic, we spent the overlay of transmitted and fluorescent channels (Fig. 3E). As a result of intensive multiphoton radiation the majority of melanocytes in the primary brain tunic observed "light reaction" in which the outflow of melanin to the central part of the cell body was recorded (Fig. 3E). Such melanocytes, devoid of outgrowths were observed through a transmitted channel. DiI-labeled cells in deep layers of the midbrain tegmentum of juvenils $O$. keta, were visualized through fluorescent channel and grouped into small conglomerates (Fig. 3E). Thus, the overlay of transmitted and fluorescent channels made it possible to reconstruct 3-dimensional picture of fluorescent cell conglomerates in the midbrain tegmentum which was located in deep layers in combination with surface patterns of distribution of melanocytes in the primary brain tunic in the mode of in vivo imaging.

\subsection{D Reconstruction}

Based on the analysis galleries of optical sections of the midbrain of juvenils $O$. keta was created 3D reconstruction of the spatial distribution of DiI-labeled cells in the damaged area of tegmentum (Fig. 3F). This 3D reconstruction reveals that the distribution of fluorescent cells, 2 days after injury to the tegmentum of juvenils $O$. keta uneven. It revealed the formation of various DiI-labeled cell conglomerates, the number and amount of which increases toward the area of injury. Thus, for the surface layers of tegmentum characterized by large clusters of DiI-labeled cells (Fig. 3F), in the deeper tegmental layers smaller clusters of fluorescent cells were localized. Study of spatial relationships of DiIlabeled cells after 30 minutes of injury indicates the predominance of large accumulation of these cells in the superficial layers of the tegmentum and their distribution in the form of small conglomerates in the deeper layers.

\section{Discussion}

The lipophilic tracer 1,1'-dioctadecyl-3,3,3'3'tetramethylindocarbocyanine perchlorate (DiI) fluorescent dye which is often used to identify morphological structure of neurons and their processes. It was introduced 25 years ago as a neuronal tracer in fixed tissue [14]. This substance and some of its derivatives have since then been widely used because of the unique possibility of labeling the neuronal connections of structures which are not easily accessible in vivo. Recently, DiI has been used both at fixed preparations of brain [15, 16], and at the living material [11]. Currently carbocyanine dyes are used not only for the dyeing of nerve cells, but also for staining of tumor cells [17]. In studies on the fixed material staining, it is accomplished by lateral diffusion of dye in lipid fractions of membrane. This is the basis for qualitative detection of myelinated paths [16]. However, the process of lateral diffusion of the dye in the membrane lipid fractions is fairly slow, from a few days to a few weeks (or months) [11]. This passive process is responsible for the long incubation period and limits the maximal distance the tracer can cover. At the same time quite often was revealed occurrence of transneuronal labeling at which revealed whole cell accumulation [18]. The identification of morphological structure of some brain nuclei and study of the cellular composition of cerebral cortex, cerebellar cortex, visual cortex is performed with this technique. Recently, it is shown that the DiI-staining has no appreciable toxic effect on the functional activity of living cells [11]. Thus, there is a real possibility to use this dye in in vivo studies.

In our studies, DiI was used as a dye for vital fluorescent multiphoton confocal microscopy. The results of in vivo monitoring in different time after injury (30 min and 2 days) suggest that as a result of midbrain injury of juvenile $O$. keta has experienced rapid cellular response, and the emergence of numerous stained cells in the injuries area. It is indicating active participation of such cells in migration and 
phagocytosis of the dye in the area of injury.
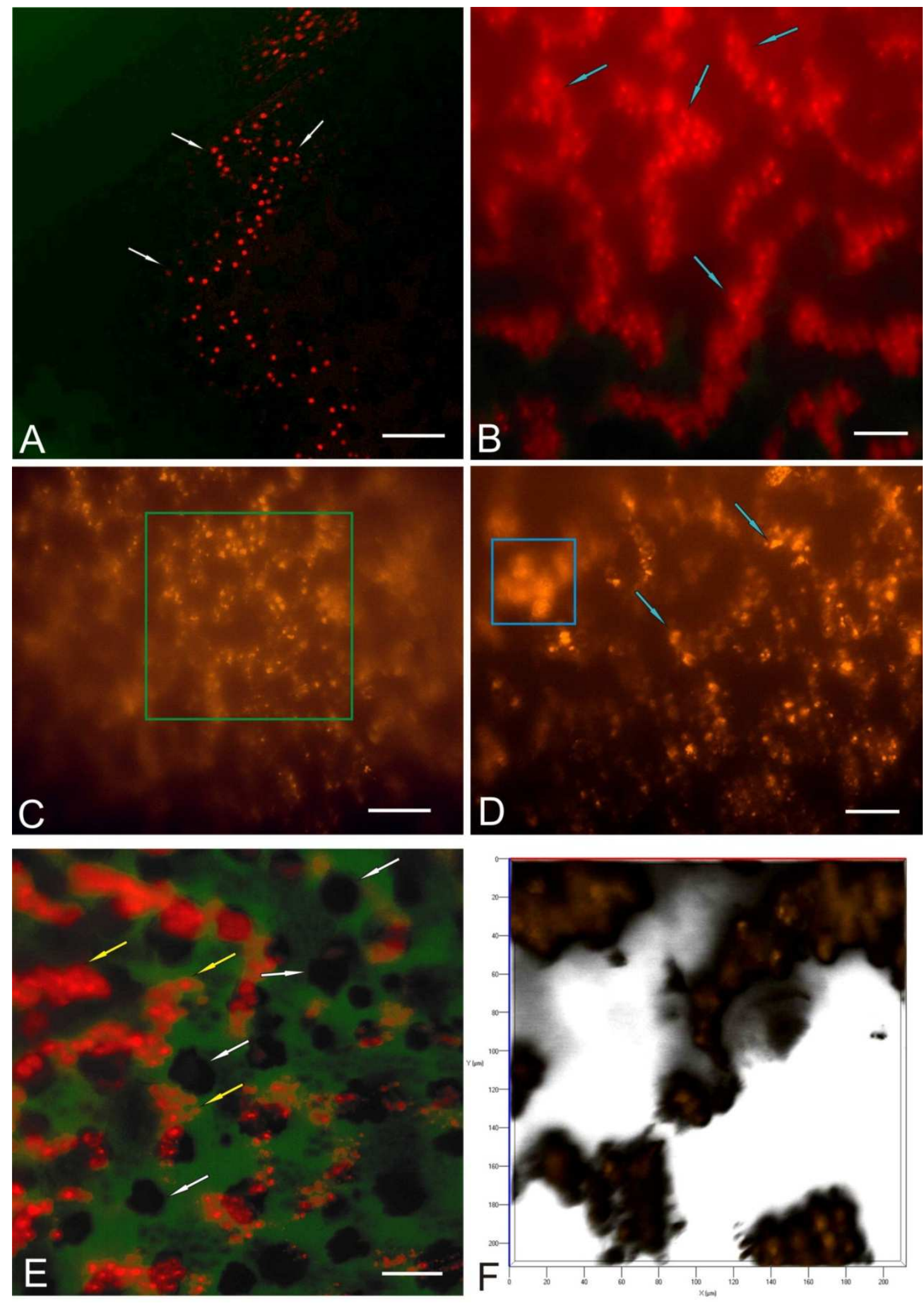

Figure 3. The results of in vivo monitoring at different times after injury of midbrain tegmentum of juvenile chum salmon Oncorhynchus keta and injection of carbocyanine dye (DiI) into the area of injury. A - DiI-labeled cells (white arrows) in the surface layers of tegmentum 30 minutes after injury; $B$ - clusters of DiI-labeled cells (blue arrows) 2 days after the injury; $C$ and D - DiI-labeled cells different types 30 minutes after injury, $C$ - cells of 2nd type in the central part of the optical section (contoured by green square), D - cells of 3rd type (blue arrows) and 4th type (contoured by blue square) in the tegmentum; Eoverlay of transmitted and fluorescent channels. Transmitted channel show body of DiI-unlabeled melanocytes (white arrows) with a "light reaction". Fluorescence channel show DiI-labeled conglomerates of cells (yellow arrows). F - 3D reconstruction of 10 optical sections DiI-labeled cells in damaged tegmentum 2 days after injury. Multiphoton confocal microscopy. A, B, D-special planar lens (magnification 20x). Scale bar: 50 um. 
Study of correlation between the parameters of DiI-labeled cells and the intensity of their fluorescence $30 \mathrm{~min}$ after injury revealed the presence of a weak correlation (Pearson coefficient 0.21). Densitometric parameters of OD in noncellular elements of 1 st type and cells of 2 nd type are almost identical (Table). This, in our opinion may indicate that small particles of dye fluoresce almost as well as dye particles captured by small cells of 2 nd type. We believe that such labeling of cells with DiI is due to their ability to phagocytize microparticles of dye injected into the area of injury. Such ability in the CNS of vertebrates is present in only two types of cells: macrophages and microglia.

The mechanism of staining of neurons by DiI is associated with the ability of dye to diffuse freely on the lipid membranes of nerve cells, marking body of nerve cells and their processes. Velocity of DiI distribution on the neuronal membrane on a fixed material depends from different components: temperature, content of certain specific ions (notably $\mathrm{Ca}^{2+}$ ) in the medium [19]. Nevertheless, in all cases, DiI-labeling of neurons takes from several hours to several days. Although this is commonly regarded as a minor problem or is not considered at all in some papers, it is observed to a variable degree in many of the studies utilizing this tracer [20-24]. Transneuronal transport of tracers is sometimes desirable, e.g. for the determination of the establishment of synapses during ontogeny [25].

In our observations elective labeling of cells was detected as early as 30 min after injury followed by injection of DiI in the injured area. We believe that such rapid and selective staining of cells in the area of injury is associated with the ability of cells to phagocytize the dye particles, and thereby achieving rapid and selective staining of cells in the zone of injury. However, among the DiI-stained cells according to morphometric analysis we found population of large cell of 4th type (3.7\%). We believe that the cells of this type can be quite sporadically stained neurons of the dorsal tegmental area of midbrain. In our observations we identified one cell with size 21.1/13.6 $\mu \mathrm{m}$, which we also refer to DiI-labeled large motoneurons located in the tegmental area of fish. The totality of these cells forms the nucleus of medial longitudinal fasciculus and the oculomotor nuclear complex situated on the territory of midbrain tegmentum. DiI-labeled cells of 4th types are corresponding to motor nuclei of tegmentum. Thus, we performed in vivo morphometric characteristics in the cellular composition, early stage of development of juvenile $O$. keta. It gave the detailed cytomorphological characteristic of living cells in a state of migration and phagocytosis.

The existence of cells with different morphological parameters can testify to the presence of different size groups of phagocytes and the various functional states of the cells in the area of trauma. Cells with different morphological characteristics may be of different cell populations of the same type. For example, during movement, cells may have a more elongated shape, which indicates the state of cell migration.
Patterns of cell conglomerates occurred in the area of injury after 2 days often matched the spatial distribution of microvascular network (Fig. 3B). According to Horsberg [26] the formation of blood brain barrier in the brain of salmon is completed by the 1 st year of life. We assume that the source of DiI-labeled cells in the perivascular space of 3-month-old juvenile chum salmon may be cerebral vascular bed. Such cells can be introduced with blood flow to the brain and provide a tissue macrophage population. In the deeper layers of the brain, sometime we identified DiI-labeled cell conglomerates containing elements that differ in terms of optical density and morphometric characteristics. The appearance of such conglomerates for a limited period of time after the application of traumatic impact to the midbrain of juveniles Oncorhynchus keta indicates the existence of rapid physiological response from the population of cells having phagocytic activity. This conclusion was obtained by us as a result of monitoring the cellular composition in the areas of trauma and the available data [19], about the speed of DiI staining of different types of cells, both in fixed and in the fresh material. On the basis of the data obtained from in vivo monitoring and morphometric analysis, we hypothesize the possibility of appearance of regional macrophages (cells of 3rd type) and intracerebral microglia (cells of 2nd type) in injury zone. After the injury was established quantitative predominance of small cells of 2 nd type (67\%) of the labeled cells in the zone of injury.

Overlay of transmitted and fluorescent channels gave a beautiful 3-dimensional picture in vivo during the study of the damaged brain of $O$. keta. We observed a lot of melanocytes located in the outer layers of the brain in primary brain tunic. After multiphoton laser irradiation most of melanocytes have typical "light reaction"; in these cells lack outgrowths and the pigment is localized in the central part of the cell body. In the foreground of this picture are present conglomerates of DiI-labeled macrophages/microglia. All this creates a new understanding of the spatial organization and structural relationships of different functional cell complexes in the brain of juvenile chum salmon after injury.

The results obtained allow the use of injection of DiI in the area of brain injury as a method to identify a population of phagocytic cells in the brain, based on the physiological response of macrophages/microglia. Thus, the injury with injection of small particles of dye DiI causes the phagocytic response from macrophages within a few minutes after the application of the damaging effects. This allows the use of DiI as a vital nonspecific marker of macrophages/microglia. Proposed method can be regarded as an effective way of identifying populations of phagocytic cells in the brain, because such methods that allow selective identification of populations of macrophages and microglia in the brain of the fish have not been developed.

We presumed that using in vivo multiphoton confocal microscopy allow us to have the substantial preference over the existing techniques. Damage to living cells decreases 
photo induced processes, because of the much lower absorption of tissues and cells in the infrared region as compared with the ultraviolet one. For this reason, it provides more depth of penetration in biological objects (animal). The main consequence of this situation is the better survival of a biological object with good image quality.

\section{Acknowledgements}

This work were supported by Program of fundamental investigation FEB RAS «Far East» 2015-2017 (grant № 15-I6-116); Grant of President of Russian Federation (MD4318.2015.4) and DST-INSPIRE Faculty Grant (IFA14LSBM-104), from the Department of Science and Technology (DST), Government of India

\section{References}

[1] Zupanc G.K. (2009). Towards brain repair: insights from teleost fish. Semin. Cell Dev. Biol., 20:683-690.

[2] Pushchina E.V., Varaksin A.A., Obukhov D.K. (2014). Participation of neurochemical signaling in adult neurogenesis and differentiation. Neurochemistry. Ed. T. Heinbockel. Rijeka, Intech, pp. 225-255.

[3] Zupanc G.K., Kompass K.S., Horschke I., Ott R., Schwarz H. (1998). Apoptosis after injuries in the cerebellum of adult teleost fish. Exp. Neurol., 152:221-230.

[4] Becker C.G., Becker T. (2008). Adult zebrafish as a model for successful central nervous system regeneration. Restor. Neurol. Neurosci., 26:71-80.

[5] Palmer A.M., Marion D.W., Botscheller M.L., Bowen D.M., DeKosky S.T. (1994). Increased transmitter amino acid concentration in human ventricular CSF after brain trauma. Neuroreport, 6:153-156.

[6] Grosche J., Härtig W., Reichenbach A. (1995). Expression of glial fibrillary acidic protein (GFAP), glutamine synthetase (GS), and Bcl-2 protooncogene protein by Müller (glial) cells in retinal light damage of rats. Neurosci. Lett., 185:119-122.

[7] Kerr J.F., Gobé G.C., Winterford C.M., Harmon B.V. (1995). Anatomical methods in cell death. Cell death. Eds. L.M. Schwartz, B.A. Osborne. San Diego, Acad. Press, pp.1-27.

[8] Zupanc G.K. Hinsch K., Gage F.H. (2005). Proliferation, migration, neuronal differentiation, and long-term survival of new cells in the adult zebrafish brain. J. Comp. Neurol., 488:290-319.

[9] Vajda F.J. (2002). Neuroprotection and neurodegenerative disease. J. Clin. Neurosci., 9:4-8.

[10] Zupanc G.K., Sirbulescu R.F. (2013). Teleost fish as a model system to study successful regeneration of the central nervous system. Cur. Top. Microbiol. Immunol., 367:193-233.

[11] Sabnis R.W. (2010). Handbook of biological dyes and stains: synthesis and industrial applications. John Wiley \& Sons. Print ISBN: 9780470407530,544 p.
[12] Stein G.I. (2007). Guide software confocal microscopy. St. Petersburg: INC RAS, 77 p.

[13] Candal E., Anadón R., DeGrip W. Rodríguez-Moldes I. (2005). Patterns of cell proliferation and cell death in the developing retina and optic tectum of brown trout. Brain Res. Devel. Brain Res., 154:101-119.

[14] Godement P., Vanselow J., Thanos S., Bonhoeffer F. (1987). A study in developing visual systems with a new method of staining neurons and their processes in fixed tissue. Development, 101:697-713.

[15] Alpeeva E.V., Makarenko I.G. (2009). Perinatal development of the mammillothalamic tract and innervation of the anterior thalamic nuclei. Brain Res., 1248:1-13.

[16] Makarenko I.G. (2008). DiI tracing is a useful tool for studies of the hypothalamic connections during perinatal development Neural pathways research. Chapter II. Ed. F.L. Pichler. NewYork, Nova Sci. Publishers, pp. 31-71.

[17] Yefimova S.L., Kurilchenko I.Yu., Tkacheva T.N., Rozhkov V.A., Sorokin A.V., Lukianova N.Y., Bezdenezhnykh N.A., Malyukin Y.V., Chekhun V.F. (2012). Comparative study of dye-loaded liposome accumulation in sensitive and resistant human breast cancer cells. Exp. Oncol., 34(2):101-106.

[18] Pushchina E.V., Karpenko A.A. (2007). Distribution of cholineacetyltransferase histochemistry in isthmus and medulla of Onchorynchus masou. Tract-tracing observation on the ascending meso-pontine cholinergic system. Tsitologiia. 49(7):581-593.

[19] Hofmann M.H., Bleckmann H. (1999). Effect of temperature and calcium on transneuronal diffusion of DiI in fixed brain preparations. J. Neurosci. Methods, 88:27-31.

[20] Striedter G.F. (1990).The diencephalon of the channel catfish, Ictalurus punctatus. II. Retinal, tectal, cerebellar and telencephalic connections. Brain Behav. Evol., 36:355-377.

[21] Striedter G.F. (1991). Auditory, electrosensory, and mechanosensory lateral line pathways through the forebrain in channel catfishes. J. Comp. Neurol., 312:311-331.

[22] Wicht H., Northcutt R.G. (1990). Retinofugal and retinopetal projections in the pactfic hagfish, Eptatretus stouti (Myxinoidea). Brain Behav. Evol., 36:315-328.

[23] Wullimann M.F., Meyer D.L., Northcutt R.G. (1991). The visually related posterior pretectal nucleus in the nonpercomorph teleost Osteoclossum bicirrhosum projects to the hypothalamus: a DiI study. J. Comp. Neurol., 312:415-435.

[24] Bartheld C.S., Cunningham D.E., Rubel E.W. (1990). Neuronal tracing with DiI: decalcification, cryosectioning, and photoconversion for light and electron microscopic analysis. J. Histochem. Cytochem., 38:725-733.

[25] Bruce L.L., Christensen M.A., Fritzsch B. (1997). Electron microscopic differentiation of directly and transneuronally transported DiI and applications for studies of synaptogenesis. J. Neurosci. Methods, 73:107-112.

[26] Horsberg T.E. (2012). Avermectin use in aquaculture. Curr. Pharm. Biotechnol., 13:1095-1102. 